\section{TATRA \\ MOUNTaiNS \\ Mathematical Publications}

DOI: $10.1515 / \mathrm{tmmp}-2015-0049$

Tatra Mt. Math. Publ. 64 (2015), 205-215

\title{
STEGANALYSIS OF STEGOSTORAGE SYSTEM
}

\author{
Michala Gulášová — MATÚš JóKAY
}

\begin{abstract}
The aim of this contribution is to detect the presence of messages in JPEG images that were stored in the files using sequential embedding into the least significant bits of DCT coefficients. The Stegostorage system was used for embedding. For testing purposes, we set this system to the full capacity of cover files as the worst case from the view of security. For steganalysis, we used and implemented a process of calibration of JPEG images, which makes it possible to investigate a histogram from steganographic images which are similar to the histogram of the original images without secret information. Also, Pearson's Chi-square test of goodness of fit was implemented, whose results indicate detectability of the presence of hidden messages through images in the two databases, the first database containing 1000 JPEG images and the second one containing 450 JPEG images. An interesting result is that after saving messages into the images and compressing the JPEG once more, the detectability decreased in this case by almost half.

Moreover, this contribution provides a framework for further testing by calculating the minimum square error (the weighted stego analysis), which also indicates detectability in both databases, and after embedding the messages into images and re-compressing them with different quantization matrix, the detectability is only slightly reduced. Therefore, we consider this method an appropriate one for further testing and utilizing it when embedding into the LSB randomly, without filling the full capacity of the carrier file.
\end{abstract}

\section{Introduction}

The need for encrypted communication is still growing. With the raise in importance of cryptography, "invisible" encrypted information becomes a more important issue, too. Only steganography can provide this "masking". It allows to hide any ongoing communication, no matter whether encrypted or not. The opposite pole of steganography is steganalysis. Its main objective is to

(C) 2015 Mathematical Institute, Slovak Academy of Sciences. 2010 Mathematics Subject Classification: 68P30, 94B05, 94A08.

Keyw ords: steganography, steganalysis, LSB embedding, JPEG, Chi-square attack.

This project is based upon work supported under the grant VEGA 1/0173/13 and is co-funded by the EEA Grant SK06-IV-01-001 and the state budget of the Slovak Republic from the EEA Scholarship Programme Slovakia. 


\section{MICHALA GULÁŠOVÁ — MATÚŠ JÓKAY}

detect such a stealthy communication. Therefore, we consider this other side of steganography as an interesting object of study.

The main focus of this contribution is the detection of messages possibly present in JPEG images, specifically the messages inserted through a system called StegoStorage [1. This system is able to hide a single message into hundreds or thousands of images, making the detection more difficult. It makes use of embedding into the least significant bit of DCT coefficients, which can be sequential, pseudo-random but can also make use of Hamming codes [1. Our aim is to achieve detectability of sequential embedding with the full capacity of filling a carrier medium.

This contribution is divided into four sections. In Section 2 we introduce the basic concepts of the steganography and briefly describe the JPEG image compression algorithm. In Section 3 we describe a scheme of StegoStorage system and its advantages over the others. In Section 4 we present two types of the attacks and our mathematical model, which is necessary for expressing the dependency of pairs of values. In the last section, we take a look at the obtained results.

\section{Preliminaries}

\subsection{Steganography}

A steganographic system is defined as a specific steganographic algorithm, which specifies the method of embedding (as well as the method of extraction). More formally in [2] it is defined as a quintuple (X, M, K, Embed, Extract) where $X$ is the set of covers transmittable over the public channel, $M$ is the set of all possible messages, $K$ is the key space, Embed is a function: $M \times X \times K \rightarrow X$ and Extract is a function: $X \times K \rightarrow M$ (note that the recipient does not need to know the original cover object to extract the message). This definition is different from the $\mathrm{Z} \mathrm{h}$ a $\mathrm{ng}$ and L i model [3], where there is a sextuple with separate domains for the covers and the stego objects.

There are the following file types in the concept of digital steganography:

- cover files are used to hide the secret message,

- carrier or stego files are cover files with hidden message.

The maximum size of the secret message that can be inserted into the cover file is the so-called capacity of the cover file. [1, [4]-[7].

\subsection{JPEG domain}

Today, we can distinguish between the types of the digital medium which are used as the cover medium. The file types include text files, image files, audio files, video files, executable files, and datagrams (or packets) of a network protocol [7]. 


\section{STEGANALYSIS OF STEGOSTORAGE SYSTEM}

In our work, we specialize in image file steganography, or more specifically, JPEG file steganography.

Let us now present a short overview of the JPEG compression algorithm and of the existing steganographic techniques that allow hiding a secret message in a JPEG cover file [1. JPEG is a lossy compression method used in various file formats.

According to [1], 7], JPEG encoding consists of the following stages:

1) Color space transformation.

2) Downsampling.

3) Block splitting.

4) Discrete cosine transform.

5) Entropy coding.

Color space transformation: it is the process of converting from RGB to $\mathrm{YCbCr}$ colour space, allowing more efficient compression without any significant effect on the perceived image quality.

Downsampling: it is the first information loss in JPEG compression. This is based on the fact that the human eye is more sensitive to brightness than to color divergence.

Discrete cosine transform: it is the process of converting an $8 \times 8$ grid of pixel color values to an $8 \times 8$ matrix of DCT coefficients.

Quantization: it is the next information loss in JPEG compression. The result of this step is the matrix with quantized DCT coefficients.

Entropy coding: it is a type of lossless compression based on the idea that the final form of a matrix with quantized coefficients consists of a large number of zeros and a couple of small integers grouped in its top left corner. It consists of two steps: creating a transition sequence of symbols, and Huffman (or arithmetic) coding.

\section{StegoStorage}

This section has been written in compliance with [1]. StegoStorage steganographic system allows to create a virtual disk over hundreds or thousands of JPEG files, unlike most other steganography applications that allow to hide secret information or a file into a single image. Thanks to that, StegoStorage has several advantages. One of them is the possibility to decide whether we would like to transmit the message parts separately or to place them in different locations. This system uses the so-called key dependent permutations. The key is formed by filenames along with the relative file paths. The key can optionally 


\section{MICHALA GULÁŠOVÁ - MATÚŠ JÓKAY}

be password-formed. Consequently, the process of extracting the secret information should require the same files and the password which were used to hide it. The system uses two types of permutations:

- global permutation-provides global diffusion (spreads bytes of hidden storage into files),

- local permutation - is permutation of LSB bits within one file (for steganalysis there is a countermeasure against the steganalysis important).

Moreover, StegoStorage exploits embedding of the secret information into LSB bits of nonzero DCT coefficients using sequential, pseudo-random embedding or Hamming codes $\left(2^{k}-1,2^{k}-k-1\right)$. These codes minimize the total number of changes in the carrier file, increasing the efficiency of embedding whilst decreasing probability of detection [1], [1]. The system architecture can be seen in Figure 1 .

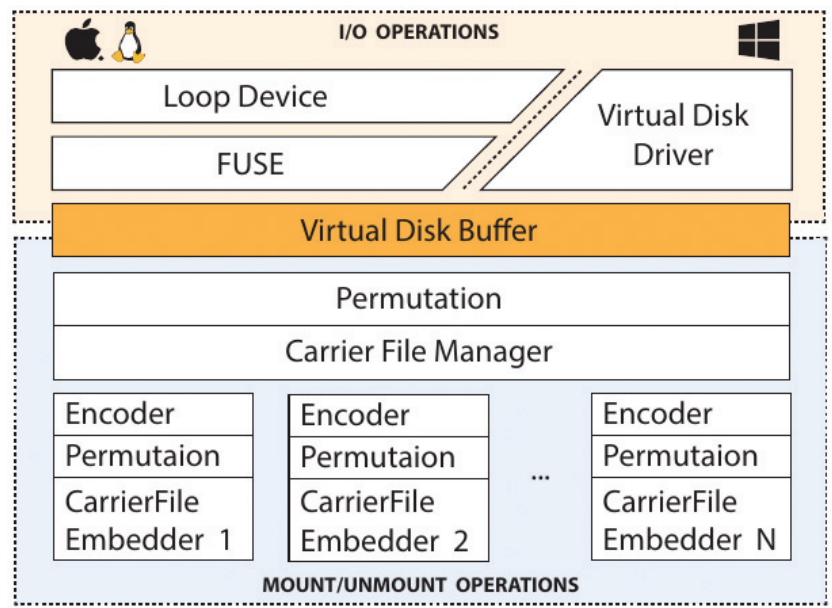

Figure 1. The architecture of the StegoStorage [1].

\section{Steganalysis}

We shall now describe the methodology for using the classical Pearson's Chi-square test of goodness of fit, for which to have a right statistical cover model is important (cover model is a null hypothesis, denot. HO). Secondly, we show J. Friedrich's mathematical model, which is the basis for our own model. Thirdly, we give a framework for the detection of LSB embedding without the need of having the carrier media capacity fully filled. 


\section{STEGANALYSIS OF STEGOSTORAGE SYSTEM}

\subsection{The Chi-square attack}

One of the results of using an embedding algorithm like LSB sequential embedding is the creation of pairs of values (POVs). In other words, as we embed a secret information into an image using an algorithm which creates POVs, the frequencies of $2 k$ and $2 k+1$ become equal or nearly so. Furthermore, it is uncommon for the frequency of DCT coefficients $2 k$ to be (nearly) equal to the frequency of DCT coefficients $2 k+1$ in a typical image with no embedded information. The Chi-square attack was designed to detect these near-equal POVs in images. The implementation of this attack is as follows [8].

Let $x^{1 \times 128}$ and $y^{1 \times 128}$ be two vectors, which $k$ th value is $x_{k}=$ frequency of $2 k$ DCT coefficients (even) and $y_{k}=$ frequency of $2 k+1$ DCT coefficients (odd), where $-64 \leq k \leq 63$. Note, that we have in our implementation of calibration a total range of DCT coefficients from -128 to 127 . Totally 256 possible values of DCT coefficients. Now, when we have sorted frequencies into even and odd parts, we can compute our theoretical expected frequency as

$$
z_{k}=\frac{x_{k}+y_{k}}{2}
$$

We thus compute a simple average of frequencies (in the global histogram) of the adjacent values of DCT coefficients. We have also reduced the number of categories (denot. $n$ ) by one half. The Chi-square statistic, with $n-1$ degrees of freedom, is then calculated

$$
\chi_{n-1}^{2}=\sum_{i=0}^{127} \frac{\left(x_{i}-z_{i}\right)^{2}}{z_{i}} .
$$

The null hypothesis is that the difference between $x_{i}$ and $z_{i}$ is very small, ideally zero. In this case Chi-square statistic is relatively small and for a non-stego image, $\chi_{n-1}^{2}$ is relatively large because $x_{i}$ should be far from $z_{i}$.

\subsection{Friedrich's model}

In the paper [9], J. Fridrich et al. presented a steganalytic method that could detect messages hidden in JPEG images using the steganographic algorithm F5. The key element of the method was estimation of the cover-image histogram from the stego-image, due to the fact, that the real attacker does not have cover files. For this purpose a well-known calibration process of JPEG images is used.

We were inspired by their analysis of histogram modifications, where they indicated the mathematical expressions of the expected values of the stego-image histograms for F5 algorithm. This algorithm is different from our algorithm, contained in StegoStorage, but using their process we were able to fit a mathematical model for our system. They also experimented with different formulas 


\section{MICHALA GULÁŠOVÁ — MATÚŠ JÓKAY}

for parameter $\beta$-the probability that a non-zero AC coefficient will be modified. The best performance they obtained using the least square approximation for this parameter. So, this approximation we implemented, too.

\subsection{StegoStorage model}

This section was written in compliance with 9 .

Let $h_{k l}(d)$ be the total number of AC DCT coefficients in the cover image corresponding to the frequency $(k, l), 1 \leq k, l \leq 8$, whose value is equal to $d$ and $d \in\{-2,-1,2,3\}$. The corresponding histogram values for the stego image will be denoted using the capital letters $H$ and $H_{k l}$. Let us assume that the LSB embedding process changes $N$ AC coefficients. The probability that a non-zero AC coefficient will be modified is $\beta=N / P$, where $P$ is the total number of non-zero AC coefficients $(P=h(-128)+h(-127)+\cdots+h(127))$.

Because the selection of the coefficients is random in StegoStorage system due to the utilization of Hamming codes, the expected values of the histograms $H_{k l}$ of the stego image are

$$
\begin{array}{ll}
H_{k l}(d)=(1-\beta) h_{k l}(d)+\beta h_{k l}(d+1), & \text { for } d=2 m \text { (even number), } \\
H_{k l}(d)=(1-\beta) h_{k l}(d)+\beta h_{k l}(d-1), & \text { for } d=2 m+1 \text { (odd number). }
\end{array}
$$

This equation expresses a dependency of pairs of values, which originates from LSB embedding. The next assumption is that we have an estimate $\hat{h}_{k l}(d)$ of the cover image histogram (acquired from the process of calibration). Now, we can calculate $\hat{H}_{k l}$ using the equation (3) and replacing $h_{k l}(d)$ by $\hat{h}_{k l}(d)$. In our calculation, we only consider four values of $d ; d \in\{-2,-1,2,3\}$, because these are the most numerous. In the [9], experiments have been carried out on how to get the value (denot. $\beta$ ), which should give the best agreement with the cover image histogram. The best results of the experiments were for the formula of $\beta$ which minimizes the square error between the stego image histogram $H_{k l}$ and the expected values of $\hat{H}_{k l}$

$$
\begin{aligned}
\beta_{k l}= & \arg \min _{\beta}\left\{\left(H_{k l}(-2)-\hat{H}_{k l}(-2)\right)^{2}+\left(H_{k l}(-1)-\hat{H}_{k l}(-1)\right)^{2}\right. \\
& \left.+\left(H_{k l}(2)-\hat{H}_{k l}(2)\right)^{2}+\left(H_{k l}(3)-\hat{H}_{k l}(3)\right)^{2}\right\} .
\end{aligned}
$$

The least square approximation in the equation (44) (differentiation with respect to $\beta$ and looking for minimum) leads to the following formula for $\beta$

$$
\begin{aligned}
\beta_{k l}= & \frac{\left[\hat{h}_{k l}(-2)-\hat{h}_{k l}(-1)\right]^{2}-\left[\hat{h}_{k l}(-2)-\hat{h}_{k l}(-1)\right]\left[H_{k l}(-2)-H_{k l}(-1)\right]}{2\left(\hat{h}_{k l}(-2)-\hat{h}_{k l}(-1)\right)^{2}+2\left(\hat{h}_{k l}(3)-\hat{h}_{k l}(2)\right)^{2}} \\
& +\frac{\left[\hat{h}_{k l}(3)-\hat{h}_{k l}(2)\right]^{2}-\left[\hat{h}_{k l}(3)-\hat{h}_{k l}(2)\right]\left[H_{k l}(3)-H_{k l}(2)\right]}{2\left(\hat{h}_{k l}(-2)-\hat{h}_{k l}(-1)\right)^{2}+2\left(\hat{h}_{k l}(3)-\hat{h}_{k l}(2)\right)^{2}}
\end{aligned}
$$

The final value of the parameter $\beta$ is calculated as the average of selected low-frequency DCT coefficients $(k, l) \in\{(1,2),(2,1),(2,2)\}$. 


\section{STEGANALYSIS OF STEGOSTORAGE SYSTEM}

\section{Results}

In previous sections, we described in more detail two types of attacks. The first attack, one of the main steganalytical attacks, is based on the fact that embedding into the least significant bits of DCT coefficients may vary in certain ways statistical properties of histograms. The second attack is based on the calculation of the probability that a non-zero AC DCT coefficient will be modified as the formula of $\beta$ which minimizes the square error between the stego images histogram and the expected values of cover images histogram acquired from the process of calibration.

We implemented all our steganalytical methods in a multi-paradigm numerical computing environment MATLAB, which is suitable for working with large matrices and lets us use a statistical toolbox.

\subsection{The Chi-square attack}

The first steganalytical method which we implemented was Pearson's Chi-square test of goodness of fit. We tested it on the standard significance level $\alpha=0.05$. In this type of statistical testing, it is important to have a correct position of the hypotheses, in other words, to have a right cover model.

We reached the best solution when we compared every second position in the global histogram of steganographic file (odd or even) with the average value of the set position and its neighbour. In this case, we relied on the fact that LSB embedding changes the global histograms in such manner that almost identical pairs of values (POVs) are formed. We thus did not need to calibrate the JPEG images here.

The results of Chi-square attack are in Tables 1 and 2, You can see in Table 1 the number of rejecting the null hypothesis, denot. HO, in the first image database consisting of 1000 images. The null hypothesis is rejected when Chi-square statistic is relatively large (this is for images without message). The equation 2 expresses the Chi-square statistic. Then we can say that after embedding the secret message into the file and re-compressing it with a different quantization matrix as before (reused JPEG decompression and compression process), 416 files were incorrectly marked as images with no message (it is the case of re-compressed stego image type).

In Table 2 you can see the number of rejecting the null hypothesis in the second image database. Unlike the previous database, our second image database consists of four hundred and fifty JPEG images, which were taken as original images in RAW format and transformed into JPEG format only once with the same quantization matrix for all images. Again we can say that 259 re-compressed stego images were marked as images without messages, although these files contained the message. 


\section{MICHALA GULÁŠOVÁ - MATÚŠ JÓKAY}

TABLE 1. The numbers of rejecting the null hypothesis in the first database of images.

\begin{tabular}{|c|c|}
\hline \multicolumn{2}{|c|}{1000 images } \\
\hline \hline The type of images & The number of rejecting the null hypothesis \\
\hline \hline stego & 0 \\
\hline no stego & 1000 \\
\hline re-compressed stego & 416 \\
\hline
\end{tabular}

TABLE 2. The numbers of rejecting the null hypothesis in the second database of images.

\begin{tabular}{|c|c|}
\hline \multicolumn{2}{|c|}{450 images } \\
\hline \hline The type of images & The number of rejecting the null hypothesis \\
\hline \hline stego & 0 \\
\hline no stego & 450 \\
\hline re-compressed stego & 259 \\
\hline
\end{tabular}

The results of this test indicate detectability throughout the entire image database consisting of 1450 files. Note, that after the re-compression of stego images with a different quantization matrix than before, the detectability decreased almost by half. Of course, after re-compression, it is impossible to recover the message from stego image. Therefore, it is unrealistic in practice an embedder(Alice) sending such images. However, this relation (between compressed and re-compressed stego image) is an important indicator in the search for a suitable model for steganalysis.

\subsection{Mathematical StegoStorage model}

The second implemented steganalytical method was the calculation of minimum squared error between the local histograms of suspicious images and their found local histograms obtained from the calibration process.

The results of testing the parameter $\beta$ are plotted in the Figures 2, 3, 4, On the $\mathrm{x}$-axis there are serial numbers of images from the database number two. On the $y$-axis there are values of the parameter $\beta$ - the probability of a non-zero $\mathrm{AC}$ coefficient modification (see Section 41). The red line shows the average value of the parameter $\beta$ of all the images in the database.

In the Figure 2, you can see the results of images with message from the second image database consisting of 450 JPEG images. The average value of the parameter $\beta$ is around 0.5 .

In the Figure 3, you can see the results of images with no message from the second image database. The average value of the parameter $\beta$ is around 0 . 


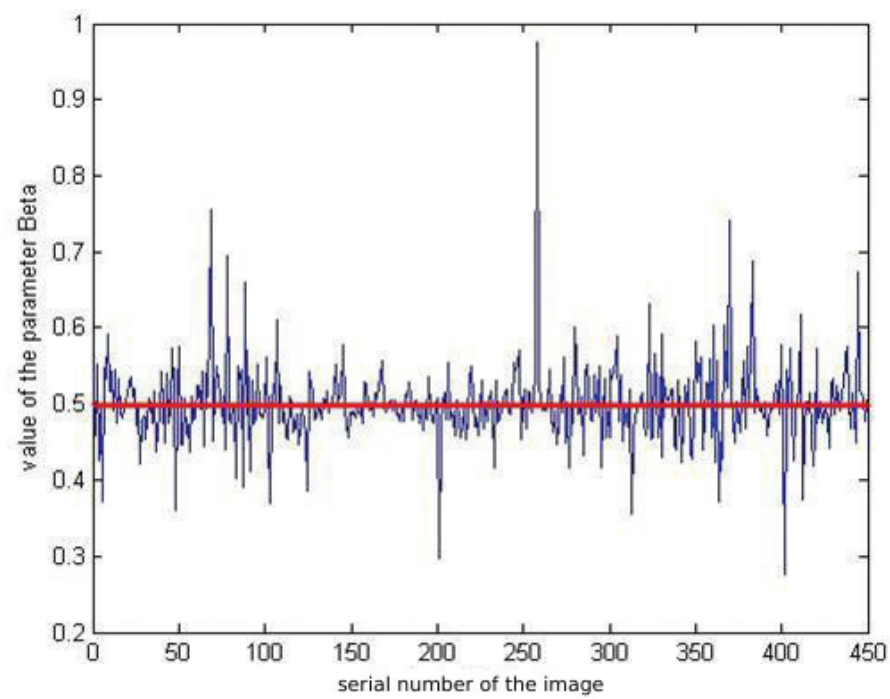

Figure 2. The images with hidden message from the database number $2=\mathrm{x}$-axis, values of the parameter $\beta=\mathrm{y}$-axis.

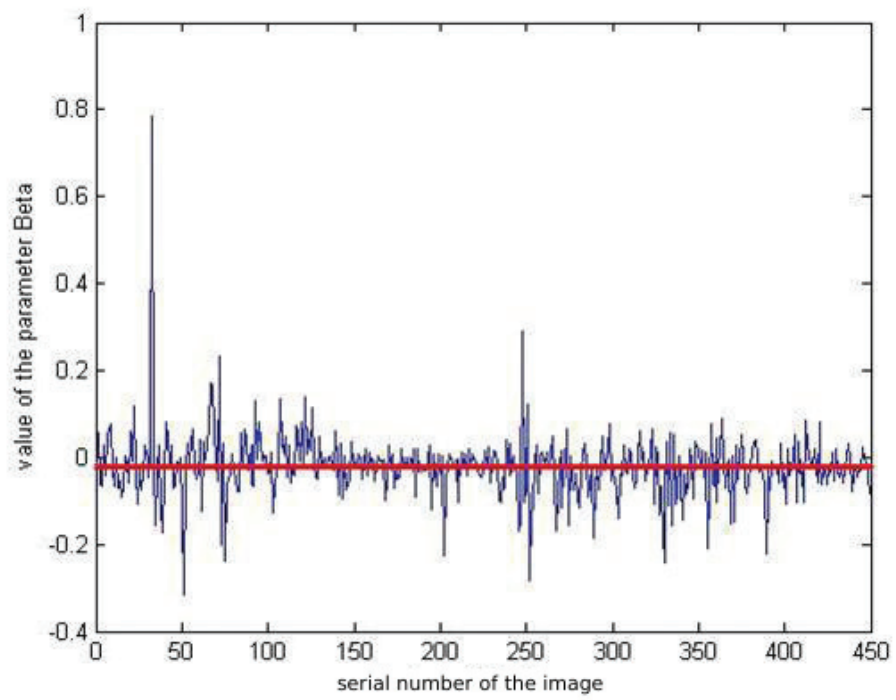

Figure 3. The images without hidden message from the database number $2=\mathrm{x}$-axis, values of the parameter $\beta=\mathrm{y}$-axis. 


\section{MICHALA GULÁŠOVÁ — MATÚŠ JÓKAY}

In the Figure 4, you can see the results of re-compressed images with message from the second image database. The average value of the parameter $\beta$ is circa 0.4 .

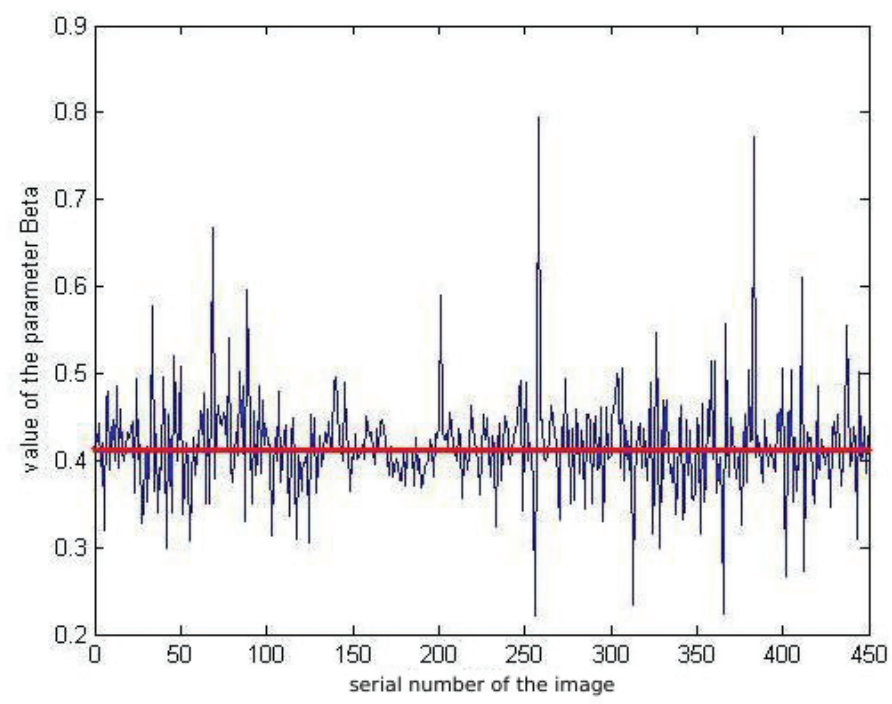

Figure 4. The re-compressed images with hidden message from the database number $2=\mathrm{x}$-axis, values of the parameter $\beta=\mathrm{y}$-axis.

In contrast to the Chi-square attack, the situation when we embedded the information into the files and re-compressed them was different. The detectability of such steganographic files decreased only slightly.

\section{Conclusion}

Analogously to the basic Chi-square attack, the results of the second attack indicate detectability throughout the entire image database consisting of 1450 files. But in contrast to the Chi-square attack, the situation when we embedded the information into the files and re-compressed them, was different. The detectability of such steganographic files decreased only slightly. This fact means that this steganalysis framework should be a more suitable approach to the detection of various types of LSB embedding than the classical Chi-square approach. So, our next goal is to find the capacity threshold for the carry files. 


\section{STEGANALYSIS OF STEGOSTORAGE SYSTEM}

\section{REFERENCES}

[1] KOŠDY, M.: Steganographic File System Based on JPEG Files: Master's Thesis. FEI STU, Bratislava, 2013.

[2] BÖHME, R.: Advanced Statistical Steganalysis. Springer-Verlag, Berlin, 2010.

[3] ZHANG, W.-LI, S.: Security measurements of steganographic systems, in: Proc. of the 2nd Internat. Conf. Applied Cryptography and Network Security-ACNS '04 (M. Jakobsson et al., eds.), Yellow Mountain, China, 2004, Lecture Notes in Comput. Sci., Vol. 3089, Springer-Verlag, Berlin, 2004, pp. 194-204.

[4] KHARRAZI, M.-SENCAR, H.-MEMON, N.: Image steganography: concepts and practice, http://www2.ims.nus.edu.sg/preprints/2004-25.pdf, 2004.

[5] CHANDRAMOULI, R.-KHARRAZI, M.-MEMON, N.: Image steganography: concepts and practice, in: Proc. of the 2nd Internat. Workshop-IWDW '03, Seoul, Korea, 2003 (T. Kalker et al., eds), Lecture Notes in Comput. Sci., Vol. 2939, Springer-Verlag, Berlin, 2004, pp. 35-49.

[6] GROŠEK, O.-VOJVODA, M.-ZAJAC, P.: Classical Ciphers. Faculty of Electrical Engineering and Information Technology, Slovak University of Technology, Bratislava, 2007.

[7] MORAVČÍK, T.: Steganografia v obrazovom sbore JPG: master's thesis. FEI STU, Bratislava, 2013.

[8] STANLEY, C.: Pairs of Values and the Chi-Ssquare Attack. Department of Mathematics, Iowa State University, 2010.

[9] FRIDRICH, J.-GOLJAN, M.-HOGEA, D.: Steganalysis of JPEG images: breaking the F5 algorithm, in: 5th Internat. Workshop-IH '02 (F. A. P. Petitcolas, ed.), Noordwijkerhout, The Netherlands, 2002, Lecture Notes in Comput. Sci., Vol. 2578, Springer-Verlag, Berlin, 2003, pp. 310-323.

[10] FRIDRICH, J.-KODOVSKÝ, J.: Calibration Revisited. ACM, New York, NY, USA, 2009.

[11] ZHANG, W.-ZHANG, X.-WANG, S.: Maximizing steganographic embedding efficiency by combining Hamming codes and Wet paper codes, in: Proc. of the 10th Internat. Workshop-IH '08 (K. Solanki et al., eds.), Lecture Notes in Comput. Sci., Vol. 5284, Springer-Verlag, Berlin, 2008, pp. 60-71.

Department of Applied Informatics and Information Technology

Faculty of Electrical Engineering and

Information Technology

Slovak University of Technology

Ilkovičova 3

SK-812-19 Bratislava

SLOVAKIA

E-mail: xgulasovam@stuba.sk matus.jokay@stuba.sk 